\title{
Brain Tumor Presenting with Parkinsonism
}

\author{
Christian Saleh Nino Akhalbedashvili Margret Hund-Georgiadis \\ REHAB Basel, Clinic for Neurorehabilitation and Paraplegiology, Basel, Switzerland
}

\section{Keywords}

Parkinsonism · Brain tumor · Neuroimaging · Diagnostic workup

\begin{abstract}
Movement disorders caused by brain tumors are rare. The diagnosis of idiopathic Parkinson's disease (PD) is based foremost on clinical findings. However, not performing imaging already within the initial diagnostic workup in patients presenting with symptoms of PD can delay or miss a serious diagnosis and consequently proper treatment. We describe and discuss a 59-year-old female patient who presented for several months of increasing tremor in her left hand, which was caused by a large meningioma located in the right frontal area, pressing on the right frontal lobe and nucleus lenticularis.
\end{abstract}

\section{Introduction}

Movement disorders caused by brain tumors are rare. The literature reports on a paucity of cases where tumors cause symptoms resembling Parkinson's disease (PD) [1-5]. Multiple tumor areas were associated with secondary parkinsonism such as the frontal, temporal, and parietal lobes, the basal ganglia, the pineal region, the posterior fossa, and the brain stem [4]. Most frequently meningiomas, gliomas, and cavernomas were associated with parkinsonism [1-5]. Parkinsonism symptoms vary, ranging from resting tremor, gait disturbance, rigidity, bradykinesia, akinesia to frozen gait [4]. The mechanisms by which tumors cause parkinsonism can be or by direct mechanical pressure on the basal ganglia nuclei, direct damage to midbrain structures, or damage to connections between the basal ganglia and the supplementary motor area [4]. 

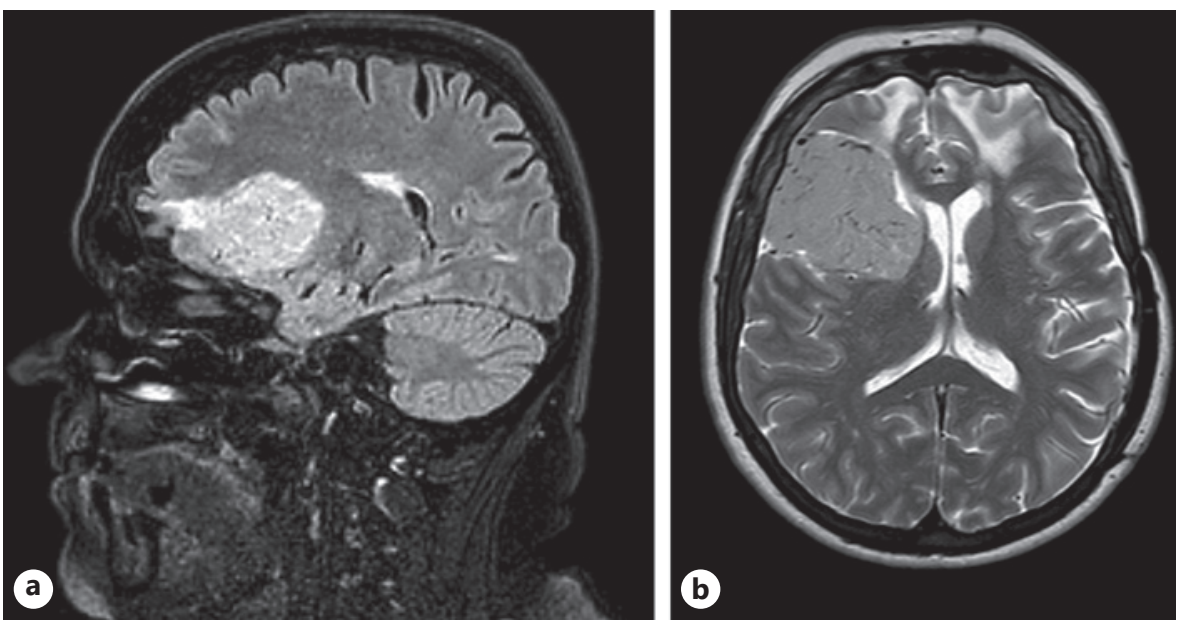

Fig. 1. a, b Sagittal (a) and axial (b) MRI images showing meningioma in the right frontal region $(4.4 \times 4.6 \times$ $4.7 \mathrm{~cm}$ ) with arterial supply via the middle meningeal artery with mechanical compression on the right frontal lobe/nucleus lenticularis. Midline displacement to the left by circa $4 \mathrm{~mm}$.

\section{Case Description}

We had a 59-year-old female patient who presented for several months of increasing tremor in her left hand. Her past medical history was remarkable for a severe traumatic brain injury with left-sided temporoparietal epidural hematoma and a metabolic syndrome. Neurological examination showed a moderate resting tremor of the left hand as of the left foot, a bilateral slight intention tremor, bradykinesia, reduced speech rate (with slight hypophonia), and a left-sided cogwheel rigidity. Pyramidal signs were absent, and sensory examination was unremarkable. On suspicion of a neurodegenerative disorder affecting the nigrostriatal system, imaging of the brain (magnetic resonance imaging and time-of-flight magnetic resonance angiography) was performed. It evidenced a large meningioma located in the right frontal area, supplied by the middle meningeal artery, pressing on the right frontal lobe and nucleus lenticularis (Fig. 1a, b). The prompt indication for a surgical intervention was made. As the tremor was not due to dopamine-deficit, we did not treat with dopaminergic medication but with a benzodiazepine (clonazepam 0.5-1.0 mg nocte). Meningioma resection was performed without complications. Postoperatively, resting tremor of the left hand diminished markedly, and only a slight tremor of the index finger was still noted at rest. Resting tremor of the left foot, bradykinesia, and intention tremor ceased fully.

\section{Discussion}

The diagnosis of idiopathic PD is based foremost on clinical findings. Neuroimaging helps to distinguish idiopathic PD from atypical or secondary forms of parkinsonism. However, despite the utility of neuroimaging, none of the different available neuroimaging techniques is routine in the initial diagnostic workup of PD [6]. In our patient, the discrepancy between tremor intensity and the paucity of other symptoms of PD raised the suspicion of a secondary parkinsonism prompting neuroimaging. We postulated that the direct mechanical effect of the tumor on the right basal ganglia structures (Fig. 1b) resulted in left-sided parkinsonism symptoms, while they subsided almost fully after tumor resection. 


\section{Conclusion}

A high degree of suspicion for secondary causes of parkinsonism is required, especially in patients presenting with atypical parkinsonism signs, poor response to anti-Parkinson medication, and focal neurological signs.

\section{Statement of Ethics}

The study is exempt from ethical committee approval. The article is a case report and as such does not need an ethical committee approval. Written informed consent was obtained from the patient for publication of this case report and any accompanying images.

\section{Conflict of Interest Statement}

The authors declare that they have no conflict of interest.

\section{Funding Sources}

The authors declare they did not receive any funding.

\section{Author Contributions}

C. Saleh, N. Akhalbedashvili, and M. Hund-Georgiadis: performed the clinical examination, wrote the draft, and revised critically the final draft.

\section{Data Availability Statement}

All data analysed for this case report are included in this article and its see online suppl. Files; for all online suppl. material, see www.karger.com/doi/10.1159/000518198. Further inquiries can be directed to the corresponding author.

\section{References}

1 Adhiyaman V, Meara J. Meningioma presenting as bilateral parkinsonism. Age Ageing. 2003;32(4):456-8.

2 Al-Janabi WSA, Zaman I, Memon AB. Secondary parkinsonism due to a large anterior cranial fossa meningioma. Eur J Case Rep Intern Med. 2019;6(4):001055.

3 Bhatoe HS. Movement disorders caused by brain tumours. Neurol India. 1999;47(1):40-2.

4 Yasuhara T, Agari T, Kambara H, Ichikawa T, Kurozumi K, Ono S, et al. Parkinsonism related to brain tumors: a case report and review of the literature. Open Neurosurg J. 2009;2(1):4-7.

5 Hollerhage M. Secondary parkinsonism due to drugs, vascular lesions, tumors, trauma, and other insults. Int Rev Neurobiol. 2019;149:377-418.

6 Pagano G, Niccolini F, Politis M. Imaging in Parkinson's disease. Clin Med. 2016;16(4):371-5.

\section{Karger'א}

